\title{
Obesidad en el niño en A mérica Latina: situación, criterios de diagnóstico y desafíos
}

\author{
O besity in Latin American children: \\ situation, diagnostic criteria and challenges
}

Hugo Amigo 1

1 Departamento de
Nutrición, Facultad de
Medicina, Universidad de
Chile. Independencia 1027,
Santiago, Chile.
hamigo@machi.med.uchile.cl

\begin{abstract}
Obesity has emerged as an alarming public health problem, having increased rapidly in both adults and children. Consi dering 12 countries with reported levels of overweight and obesity, one third have a $20 \%$ preval ence of one or both conditions. In 17 countries reporting data on obesity, the mean rate was $4.6 \%$; four countries reported rates over $6 \%$, while three reported less than $2 \%$. The majority of these countries showed increases of $70 \%$ in overweight and $60 \%$ in obesity during the last decade. Only two countries reported decreasing preval ence rates. Countries may be classified in four groups: (1) low or even downward prevalence of overweight and obesity; (2) underweight reported simultaneously with an increase in obesity; (3) a large territory and population with differences in the preval ence of overweight and obesity between regions and socioeconomic groups; and (4) little underweight and high prevalence of obesity. In conclusion, there is no doubt about the increasing trend in overweight and obesity among Latin American children. However, since there is no consensus as to diagnostic criteria for overweight and obesity, the real prevalence is not known.
\end{abstract}

Key words Obesity; Anthropometry; Nutritional Status

Resumen En la actualidad, la obesidad constituye un problema de Salud Pública y ha aumentado en adultos y niños. De 12 países latinoamericanos que tenían información completa de sobrepeso y obesidad al go más de un tercio supera el $20 \%$ en ambas situaciones. Para obesidad 17 países contaban con información; el promedio de ésta fue 4,6\%, en el que 4 de ell os presentaron valores sobre $6 \%$ y 3 inferiores al 2\%. En la mayoría se observó incremento en los últimos años, el $70 \%$ presentó aumento de sobrepeso y el $60 \%$ de obesidad, sól o en dos de ell os hubo descenso. Esta situación permite clasificar a los países en 4 grupos: el primero los que presentan bajos valores de exceso de peso y tendencia al descenso; el segundo los que poseen altos val ores de déficit de peso y aumento de obesidad; el tercero son los que tienen gran extensión territorial, en que se observa diferenciación geográfica y poblacional en el exceso de peso; y finalmente los que presentaron disminución del déficit y un importante aumento del exceso de peso. En conclusión, no hay dudas sobre la tendencia al aumento del sobrepeso y la obesidad en el niño, sin embargo, debido a la falta de consenso sobre los criterios diagnósticos los reales nivel es de prevalencia están en discusión.

Palabras clave Obesidad; Antropometría; Estado Nutricional 


\section{Introducción}

Hay un reconocimiento que el exceso de peso constituye un problema de Salud Pública (WHO, 1998). Esta afirmación ha sido realizada a pesar de existir una información limitada, especialmente en relación a la situación de los niños.

La obesidad constituye un problema relevante por los altos costos que se derivan de su atención y porque representa un factor de riesgo de varias afecciones crónicas que son de importancia para la Salud Pública. A su vez diferentes estudios señal an que existe una significativa correlación entre el peso en la niñez con el de la vida adulta (Burrows, 2000).

Actualmente, no existe claridad sobre la situación del sobrepeso y obesidad en los países en vías de desarrollo, incluidos muchos de Latinoamérica. Una interrogante a resolver es determinar si las prevalencias observadas constituyen un problema de salud pública o son especulaciones derivadas de la situación que presentan los países de mayor desarrollo. Esta afirmación se fundamentaría en una proyección casi lineal de la llamada transición epidemiológica, sin considerar la dinámica de las correcciones.

Otro elemento que se debiera abordar es conocer si la situación es homogénea en los diversos países de América Latina o varía de acuerdo a las etapas de la transición epidemiológica o por el nivel de desarrollo económico alcanzado. Lo anterior determinaría si en algunas áreas geográficas estaríamos frente a una epidemia de obesidad o sería más apropiado señalar que se trata de una endemia localizada o un simple cambio temporal en la tendencia. Estas son algunas interrogantes a abordar.

Los elementos antes planteados serán discutidos considerando los factores etiológicos que están vinculados a la situación observada, levantando algunas hipótesis sobre los posibles elementos causal es que podrían estar originando los escenarios encontrados.

La situación en el continente debiera permitir especular sobre el pronóstico para los próximos años y los desafíos que ellos implican para la formulación de intervenciones. En ese sentido se debería establecer una estrategia coherente que seleccione adecuadamente los beneficiarios y defina contenidos para programas destinados a reducir los problemas derivados del déficit $y / 0$ exceso.

Se debe mencionar que este manuscrito ha sido elaborado con la información existente, especialmente la que está disponible en las bases de datos de informaciones bibliográficas. Se ha privilegiado las provenientes de revistas especializadas e informes técnicos de agencias internacionales. Se debe aclarar que hay varios ejemplos del caso chileno donde el autor de este artículo desarrolla su trabajo profesional, sin embargo, la perspectiva de esta revisión siempre ha sido latinoamericana.

El propósito de este trabajo es señalar cuál es la prevalencia del sobrepeso y obesidad del niño latinoamericano, alguno de su factores etiológicos y los desafíos que deberán enfrentarse para prevenir la obesidad infantil.

\section{Consideraciones metodológicas}

Se ha decidido analizar la situación del sobrepeso y obesidad, porque es difícil separarlos ya que los trabajos que comunican las prevalencias o tendencias o los que analizan elementos causales, lo hacen en conjunto sin diferenciar los dos niveles. En este documento el criterio diagnóstico utilizado es el que utiliza la Organización Mundial de la Salud (OMS) porque la mayoría de los datos disponibles están analizados de esta forma así se ha considerado sobrepeso aquel aumento de peso para la estatura entre +1 y +2 desviaciones estándares y obesidad sobre dos desviaciones estándar de las referencia Internacional NCHS/ OMS (WHO, 1983).

Las fuentes de informaciones corresponden a encuestas nacionales realizadas en América Latina y analizadas por Martorell et al. $(1998,2001)$. La última actualización de la base de datos que publica la OMS y que reúne información de 160 países (Onis \& Blössner, 2000), tiene como universo el $94,1 \%$ de la población menor de cinco años del continente. Asimismo, se complementó con informaciones específicas de muestras o censos nacionales de Chile (Kain et al., 2002), de Brasil (Monteiro et al., 2000) y de México (Rivera-Dommarco et al., 2001).

\section{Diagnóstico (prevalencias y tendencias)}

El consolidado de estos trabajos indica que de los 12 países de América Latina que tenían información completa de sobrepeso y obesidad algo más de un tercio supera el $20 \%$ en ambas situaciones, destacándose la alta frecuencia encontrada en niños bolivianos, peruanos y chilenos, observando que los que presentan los menores niveles son los países centroamericanos (Figura 1). Con respecto a la obesidad 17 países presentaban esta información, los valores promedios indican que es ligeramente su- 
perior al 4,5\% siendo que en cuatro de ellos se informan prevalencias sobre el $6 \%$ siendo los niños chilenos los que presentan las mayores tasas con 7,8\% (Figura 2).

Con la base de datos publicada por la OMS y actualizadas con datos chilenos y mexicanos se puede constatar la situación del continente separado por subregiones comparándolos con el africano y el asiático observándose que los mayores niveles de prevalencia se concentran en América del Sur, donde también existen los menores índices de emaciación (déficit mayor a dos desviaciones estándar de la relación peso para talla) (Tabla 1).

Para estimar tendencias se deben tener medidas repetidas en poblaciones semejantes. Así, se analizaron las variaciones en los países que tenían muestras nacionales y que las hu-

\section{Figura 1}

Prevalencia de sobrepeso y obesidad en países de América Latina en niños menores de 5 años.

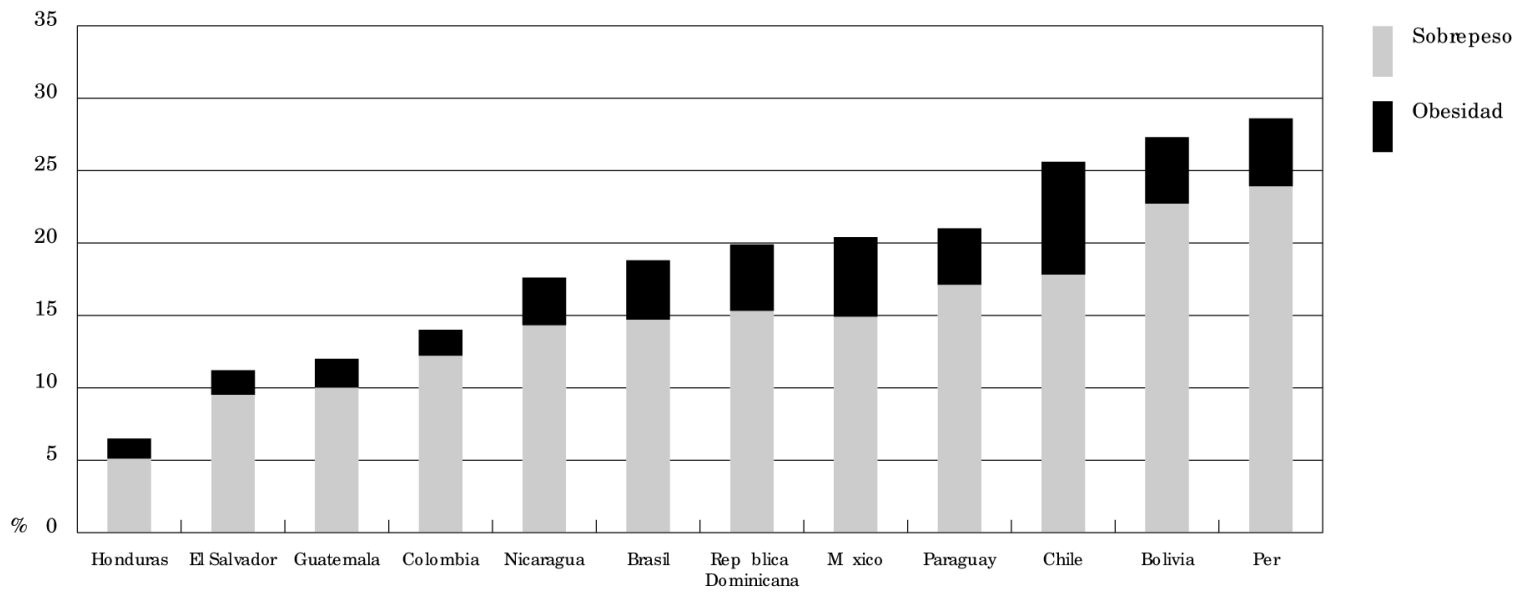

Figura 2

Prevalencia de obesidad en países de América Latina en niños menores de 5 años.

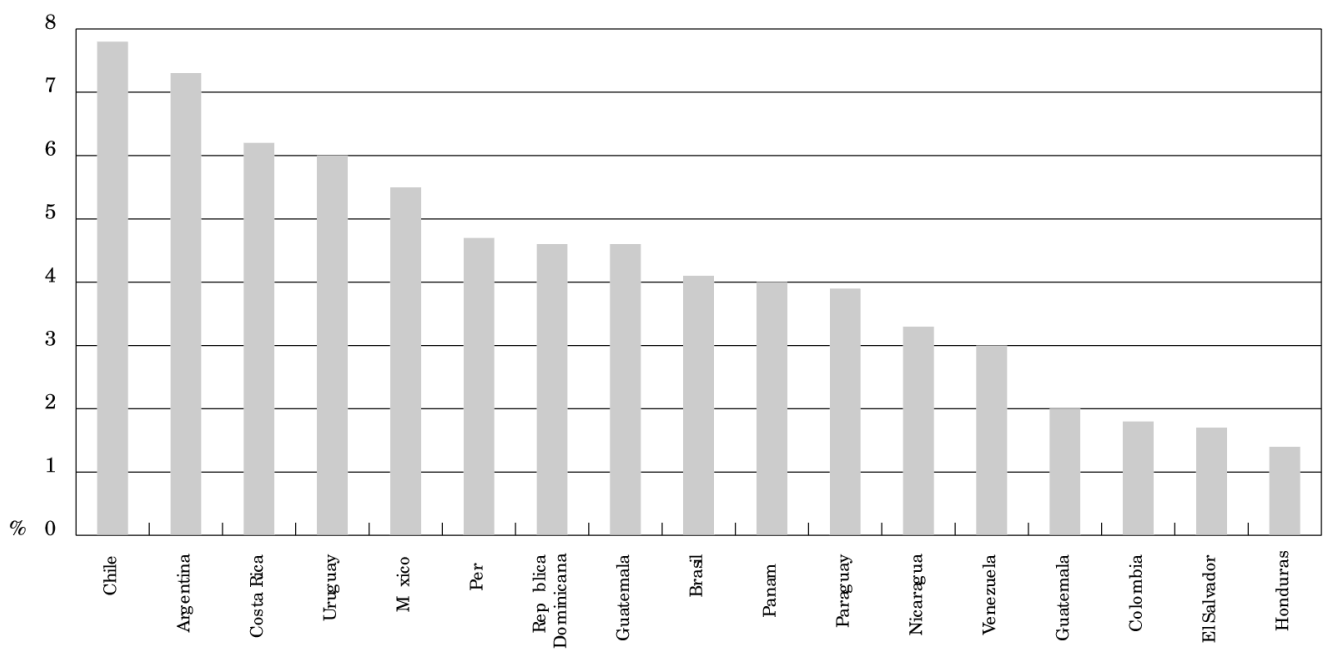


Tabla 1

Prevalencias de sobrepeso y obesidad en niños menores de cinco años en América Latina, África y Asia en 1995.

\begin{tabular}{lcc}
\hline Subregiones & Obesidad (\%) & Emaciación (\%) \\
\hline América Central & 3,5 & 4,9 \\
América del Sur & 4,6 & 1,8 \\
África & 3,9 & 9,6 \\
Asia & 2,9 & 10,4 \\
\hline
\end{tabular}

Obesidad: Exceso de peso superior a dos desviaciones estándar.

Emaciación: Déficit superior a dos desviaciones estándar. llegando a prevalencias de sobrepeso superiores a $20 \%$. En relación a la obesidad la prevalencia del último año analizado alcanza valores muy altos, sobre el $17 \%$ en hombres y casi $19 \%$ en mujeres observándose también un aumento significativo, progresivo y constante con porcentajes de incremento sobre el $10 \%$ en ambos sexos (Tabla 2).

\section{Criterios diagnósticos}

Se ha indicado que los cambios observados de sobrepeso y obesidad podrían deberse a modificaciones de criterios diagnósticos o al uso en algunas oportunidades de una referencia internacional y en otras locales.

Con respecto a los criterios diagnósticos hasta la década de los ochenta el más utilizado era el porcentaje sobre la mediana, la mayoría de los países definían sobrepeso como aquella adecuación entre 110 y $120 \%$ y sobre $120 \%$ para el caso de la obesidad. Probablemente esta forma de expresión estuvo influenciada porque el déficit de peso era referido de esta forma o por las facilidades para realizar este tipo de cálculos. Con el uso progresivo de la informática, la utilización de las medidas de dispersión han sido las de mayor uso (WHO, 1995). También en aquel tiempo existía una cierta heterogeneidad de uso de patrones antropométricos en los países de Latinoamérica y algunos de ellos construyeron sus propias referencias como es el caso de Brasil que utilizó la de una ciudad del Estado de São Paulo, Santo André (Marques et al., 1974) y en el caso chileno se utilizó la referencia francesa de Sempé (1984) y los países de Centroamérica la de IOWA (Stuart \& Stevenson, 1954, apud Nelson, 1959).

Existen pocos antecedentes de la comparación utilizando diferentes referencias antropométricas o definiciones del sobrepeso u obesidad. Recientemente un estudio chileno antes mencionado comparó distintos criterios diagnósticos concluyendo que efectivamente hay cambios en los niveles de prevalencia, aunque no de la tendencia observada (Kain et al., 2002).

Los criterios de mayor uso para clasificar sobrepeso y obesidad en el niño son los siguientes:

- El recomendado por el comité norteamericano de expertos para la obesidad del niño (Barlow \& Dietz, 1998; Flegal et al., 2001) que definió como niño con sobrepeso a los que tienen un índice de masa corporal con el percentil entre 85 y 95 - según edad y sexo - y sobre el 95 para definir obesidad usando como referencia la del NCHS/OMS. 


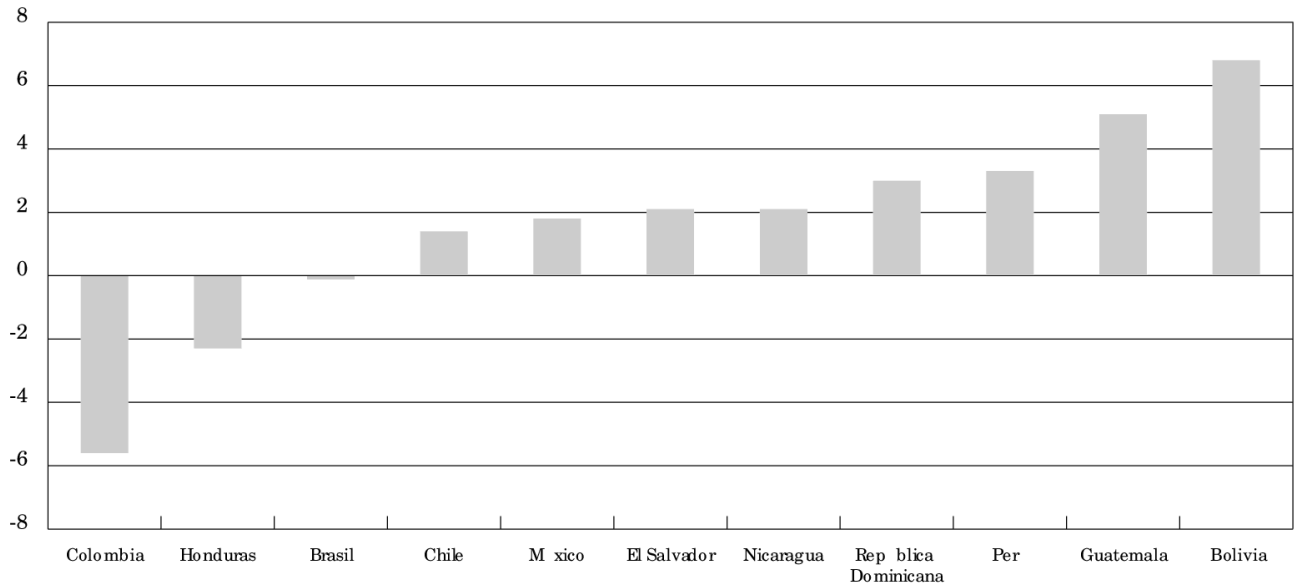

- El criterio sugerido por el grupo de trabajo internacional sobre obesidad (IOTF - International Obesity Task Force) que propuso un punto de corte proyectado de un índice de masa corporal (IMC) del adulto de 25 para el sobrepeso e igual o superior a 30 para definir obesidad (Cole et al., 2000). La referencia utilizada está basada en seis bases de datos de diferentes países. Este criterio es el que identificaría un menor número de niños con exceso de peso, sin embargo no se puede indicar que se trata de un criterio de menor sensibilidad porque la definición de la referencia oro (gold estándar) o la precisión del verdadero punto de corte para definir exceso de peso u obesidad es todavía un tema abierto.

- El criterio de mayor uso y que ha venido utilizando la OMS es la que ha definido sobrepeso a los niños clasificados entre una y dos desviaciones estándares y obesidad sobre dos desviaciones estándares del patrón antropométrico internacional (NCHS/OMS). Este criterio está basado en una distribución normal de la relación peso para talla, lo que no parece ser la que siguen nuestras poblaciones. Esta clasificación que fue la utilizada en este trabajo, implica que hay una expectativa teórica de prevalencias esperadas y este es un elemento a considerar para no incluir falsos positivos ni negativos en las estimaciones de prevalencias.

En nuestra opinión urge consensuar los criterios diagnósticos de exceso de peso y obesidad en el niño y esto debería ser una prioridad por el incremento de sus prevalencias en dife-
Tabla 2

Prevalencia y variación del incremento de exceso de peso y obesidad en escolares chilenos que ingresan en la escuela.

\begin{tabular}{lccrr}
\hline \multirow{2}{*}{ Años } & \multicolumn{2}{c}{$\begin{array}{c}\text { Exceso de peso (\%) } \\
\text { Niños }\end{array}$} & Niñas & \multicolumn{2}{c}{$\begin{array}{c}\text { Obesidad (\%) } \\
\text { Niños }\end{array}$} & Niñas \\
\hline 1987 & 15,0 & 17,2 & 6,5 & 7,8 \\
1990 & 17,3 & 19,7 & 8,9 & 10,1 \\
1993 & 18,2 & 20,7 & 11,4 & 12,7 \\
1996 & 19,0 & 21,4 & 13,4 & 15,0 \\
2000 & 20,0 & 21,8 & 17,0 & 18,8 \\
Variación del incremento & $+5,0$ & $+4,6$ & $+10,1$ & $+11,0$ \\
\hline
\end{tabular}

rentes latitudes. Para definir estos criterios, hay que tener en cuenta la validez del índice de masa corporal y la utilización de una adecuada referencia internacional.

Con respecto a la validez se ha indicado que los puntos de corte para evaluar exceso de peso y obesidad en el adulto basados en el IMC no tienen una buena concordancia con la proporción de grasa y masa magra (Himes et al., 1991) y que estos factores varían con la edad y el sexo del niño(Lazarus et al., 1996), sin embargo, para estudios poblacionales son de utilidad. En relación a la referencia internacional existe una progresiva discusión sobre la necesidad de una definición de consenso para al respecto (Chinn \& Rona, 2002). Esta referencia debería ser elaborada considerando antece- 
dentes socioeconómicos, culturales y una situación de normalidad en relación a los niveles de prevalencia. Se debe tener cuidado que la referencia internacional elaborada no sea el promedio de realidades diferentes que terminan por no corresponder a ninguna de ellas. La referencia ideal debería ser elaborada en investigaciones bien diseñadas realizadas en lugares que no tengan ni bajas ni altas prevalencias de exceso de peso que podrían aumentar o disminuir la verdadera situación donde esta fuera aplicada.

\section{Discusión}

Los datos aquí presentados indican que en el niño habría un incremento del sobrepeso y obesidad en la mayoría de los países de América Latina y esto debe ser motivo de preocupación por la fuerte asociación con obesidad en el adulto, hipertensión, dislipedemias, enfermedades respiratorias y desórdenes psicosociales (Gutin et al., 1990; Parra et al., 1971).

Las prevalencias observadas como promedio regional no son altas, siendo algo mayor de lo esperado. Un análisis cuidadoso indica que la situación en América Latina tiene las siguientes características:

- Países en que no se observa un incremento del exceso de peso y se mantienen tasas relativamente altas de déficit de crecimiento, este sería el caso de Colombia y especialmente Honduras.

- Países en que se ha observado una tendencia al incremento del exceso de peso y obesidad, y permanecen altos índices de emaciación y déficit de crecimiento, ejemplo de esta situación es Perú, Bolivia, Nicaragua y Guatemala. Sin embargo, en estos países el incremento de la proporción de niños con estas características no supera lo esperado.

- Países de gran población y extensión territorial en que se observa un incremento del exceso de peso pero una desigualdad en su prevalencia por regiones y nivel socioeconómico. Ejemplos: Brasil y México.

- Países en que se observa una nítida tendencia hacia el aumento de los valores de exceso de peso en diferentes edades y niveles socioeconómicos, el caso más evidente es el chileno.

Sobre los países del primer grupo se debe mencionar que Honduras es uno de los que presenta mayores tasas de pobreza y los más bajos índices de salud en los niños. En el caso de Colombia, a pesar de tener un nivel de desarrollo intermedio, presenta una tendencia a la disminución de la obesidad, desafortunada- mente las informaciones disponibles no permiten concluir en forma definitiva sobre la realidad de este país y los posibles factores que estarían determinando esta situación.

Los países del grupo 2 parecen estar enfrentando una rápida transición epidemiológica en que coexisten deficiencias nutricionales y exceso de peso. En estos países deben realizarse esfuerzos para mejorar el crecimiento de los niños y a su vez es imprescindible monitorear el exceso de peso con la finalidad de tomar medidas preventivas para corregir oportunamente el problema. En otras palabras hay que evitar que el brote epidémico se transforme en epidemia.

En los países que tienen un extenso territorio, como México y Brasil, es necesario la desagregación geográfica de la información e idealmente por indicadores socioeconómicos. Afortunadamente estos países han realizado encuestas nacionales que han permitido este tipo de análisis. Con la información de Brasil se han realizado interesantes trabajos utilizando variados niveles de desagregación (Monteiro et al., 1995, 2000) y en el caso de México acaba de ser publicada su Segunda Encuesta Nacional de Nutrición, donde se puede observar la distribución de los problemas nutricionales por grandes zonas geográficas y grupos de edad (Rivera-Dommarco et al., 2001). Este tipo de información puede ser de utilidad para la elaboración de una estrategia coherente de prevención y/ o tratamiento de la obesidad infantil, dirigiendo las intervenciones a diferentes tipos de población.

El caso de Chile que ha presentado un sostenido incremento del exceso de peso en distintos grupos de edad, permite definir la situación como una verdadera epidemia y como la reversibilidad puede involucrar a más de una generación, probablemente este país enfrente los próximos años una endemia de exceso de peso en la niñez. Una de las causas de esta situación puede ser el importante aumento del Producto Geográfico Bruto y una sostenida disminución de la pobreza (Chilean Ministry of Planning, 1999), esto ha significado un cambio cualitativo de la dieta y una disminución de la actividad física lo que puede explicar en parte esta situación. A su vez ha desaparecido el déficit de peso y el retardo de crecimiento hasta casi alcanzar la distribución observada en el patrón de referencia de la OMS (Uauy \& Castillo, 2001). Así, en este país se han cumplido las metas propuestas en relación a los indicadores nutricionales por déficit, pero se ha fracasado en las relacionadas con el exceso de peso lo que debe significar una alerta para todos los 
países que tengan una rápida transición epidemiológica. Lo ocurrido en Chile probablemente es el producto del seguimiento de otros modelos de desarrollo, sin mayores correcciones incluida la falta de adecuación de los programas nutricionales.

\section{Sugerencias}

Para enfrentar los desafíos destinados a revertir el incremento de exceso de peso y obesidad en el niño se debería actuar sobre los siguientes aspectos:

- Corrección de los objetivos de los programas nutricionales lo que significa que los complementos alimentarios, que están destinados a cubrir la brecha de alimentación en muchas oportunidades constituyen un incremento de la ingesta alimenticia sobre los requerimientos nutricionales. De esta manera no es de extrañar que en muchos lugares los programas alimentarios se hayan transformado en un sistema involuntario de aumento de la obesidad en el niño. En estos lugares la estrategia de los programas alimentarios debe ser modificada por una de focalización en que las intervenciones estén dirigidas a beneficiarios considerando características culturales, socioeconómicas y nutricionales.

- Establecer actividades de Promoción de la Salud un ejemplo de estas acciones es la elaboración de guías alimenticias a ser utilizadas masivamente en programas educacionales y de salud.

- Aumento de la actividad física en ese sentido vale la pena mencionar que en algunos países desarrollados se ha recomendado realizar una actividad física moderada de 30 minutos tres días a la semana y esto debiera ser considerado en las currículos educacionales.

- Como marco de todo lo anterior se sugiere que los países establezcan estrategias nacionales de vida saludable desde la niñez, en el cual el cambio de dieta y la actividad física son acciones fundamentales. El objetivo de estas estrategias esta basada en el concepto que es necesario trabajar con la población lo más precozmente posible.

\section{Referencias}

BARLOW, S. \& DIETZ, W., 1998. Obesity evaluation and treatment: Expert committee recommendations. Pediatrics, 102:E29-E36.

BURROWS, R., 2000. ¿Existe realmente una asociación entre la obesidad infantil y la del adulto? In: Obesidad un Desafío Pendiente (C. Aballa, J. Kain, R. Burrow \& E. Diaz, org.), pp. 284-285, Santiago: Editorial Universitaria.

CHILEAN MINISTRY OF PLANNING, 1999. Poverty and Income Distributions in Chile 1998. Results from the National Socioeconomic Characterization Survey. Santiago: Chilean Ministry of Education.

CHINN, S. \& RONA, R., 2002. International definitions of overweight and obesity for children: A lasting solutions? Annals of Human Biology, 29:306-313.

COLE, T. J.; BELLIZZI, M. C.; FLEGAL, K. M. \& DIETZ, W. H., 2000. Establishing a standard definition for child overweight and obesity worldwide: International survey. BMJ, 320:1240-1243.

FLEGAL, K. M.; OGDEN, C. L.; WEI, R.; KUCZM ARSKI, R. L. \&JOHNSON, C. L., 2001. Prevalence of overweight in US children: Comparison of US growth charts from CDC with other reference values for body mass index. American Journal of Clinical Nutrition, 73:1086-1093. 
GUTIN, B.; BASCH, C.; SHEA, S.; CONTENTO, I.; DeLOZIER, M.; RIPS, J.; IRIGOYEN, M. \& ZYBERT, P., 1990. Blood pressure, fitness and fatness in 5- and 6-years-old children. JAMA, 264:1123-1127.

HIMES, J. H.; BOUCHARD, C. \& PHELEY, A. M., 1991. Lack of correspondence among measures identify the obese. American Journal of Preventive Medicine, 7:107-111.

KAIN, J.; UAUY, R.; VIO, F. \& ALBALA, C., 2002. Trends in overweight and obesity prevalence in Chilean children: Comparison of three definitions. European Journal of Clinical Nutrition, 56:200-204.

LAZARUS, R.; BAUR, L.; WEBB, K. \& BLYTH, F., 1996. Body mass index in screening for adiposity in children and adolescents: Systematic evaluation using receiver operating characteristics curves. American Journal of Clinical Nutrition, 63:500506.

MARQUES, M. M.; BERQUO, E.; YUNES, J. \& MARCONDES, E., 1974. Crescimiento de crianças brasileiras: Peso e altura segundo idade e sexo. Influenca de fatores sócio-económicos. Anais Nestlé, 84(Sup. 2):11.

MARTORELL, R.; KETTEL-KHAN, L.; HUGHES, M. L. \& GRUM MER-STRAWN, L., 2001. Obesity in children from developing countries: A public health problem? In: Nutrition and Growth (R. Martorell $\&$ F. Haschke, ed.), Nestlé Nutrition Workshop Series Pediatric 49, pp. 351-373, Philadelphia: Lippincot Williams \& Wilkins.

MARTORELL, R.; KHAN, L. K.; HUGHES, M. L. \& GRUMMER-STRAWN, L. M., 1998. Obesity in Latin American women and children. Journal of Nutrition, 128:1464-1473.

MONTEIRO, C. A.; BENICIO, M. H. D'A.; CONDE, W. L. \& POPKIN, B. M., 2000. Shifting obesity trends in Brazil. European Journal of Clinical Nutrition, 54:342-346.

MONTEIRO, C. A.; MONDINI, L.; DE SOUZA, A. L. \& POPKIN, B. M., 1995. The nutrition transition in Brazil. European Journal of Clinical Nutrition, 49:105-113.
NELSON, W. E. (ed.), 1959. Textbook of Pediatrics. 7th Ed. Philadelphia: Sanders.

ONIS, M.\& BLÖSSNER, M., 2000. Prevalence and trends of overweight among pre-school children in developing countries. American Journal of Clinical Nutrition, 72:1032-1039.

PARRA, A.; SCHULTZ, R. B.; GRAYSTONE, J. E. \& CHEEK, D. B., 1971. Correlative studies in obese children and adolescents concerning body composition and plasma insulin and growth hormone levels. Pediatric Research, 5:605-613.

RIVERA-DOM M ARCO, J.; SHAM AH-LEVY, T.; VILLALPANDO-HERNANDEZ, S.; GONZALEZ-DE COSSIO, T.; HERNANDEZ-PRADO, B. \& SEPULVEDA, I., 2001. Encuesta Nacional de Nutrición 1999: Estado Nutricional de Niños y Mujeres en México. México, DF: Instituto Nacional de Salud Pública.

SEMPE, M., 1984. Surveillance de la croissance de I'enfant. References pratiques. Le Concours Medical, Sup.: 43.

UAUY, R. \& CASTILLO, C., 2001. Nutrición de los niños en Chile: Dónde estamos, hacia adónde vamos. Revista Chilena de Pediatría, 72:1-5.

WHO (World Health Organization), 1983. Measuring Change in Nutritional Status: Guidel ines for Assessing the Impact of Supplementary Feeding Programmes for Vulnerable Groups. Geneva: WHO.

WHO (World Health Organization), 1995. The U sed and Interpretation of Anthropometry. Report of WHO Expert Committee, Technical Report Series 854. Geneva: WHO Expert Committee on Physical Status.

WHO (World Health Organization), 1998. Obesity: Preventing and Managing. The Global Epidemic. Report of a WHO Consultation on Obesity. Geneva: WHO.

Recibido el 27 de mayo de 2002

Versión final presentada el 10 de enero de 2003

Aprobado el 17 de enero de 2003 